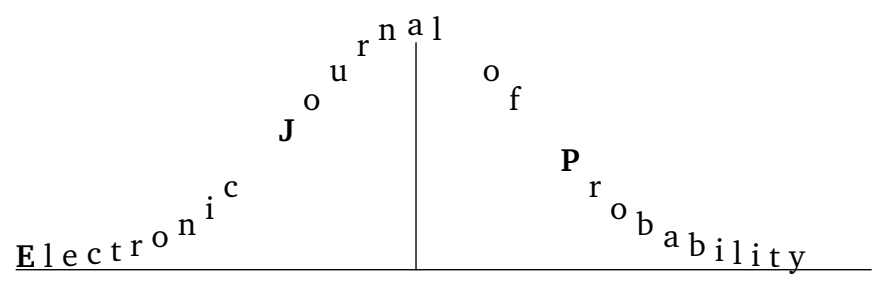

Vol. 13 (2008), Paper no. 63, pages 1927-1951.

Journal URL

http://www.math.washington.edu/ ejpecp/

\title{
A special set of exceptional times for dynamical random walk on $\mathbb{Z}^{2}$
}

\author{
Gideon Amir* $\quad$ Christopher Hoffman ${ }^{\dagger}$
}

\begin{abstract}
Benjamini, Häggström, Peres and Steif introduced the model of dynamical random walk on $\mathbb{Z}^{d}$ [2]. This is a continuum of random walks indexed by a parameter $t$. They proved that for $d=3,4$ there almost surely exist $t$ such that the random walk at time $t$ visits the origin infinitely often, but for $d \geq 5$ there almost surely do not exist such $t$.

Hoffman showed that for $d=2$ there almost surely exists $t$ such that the random walk at time $t$ visits the origin only finitely many times [5]. We refine the results of [5] for dynamical random walk on $\mathbb{Z}^{2}$, showing that with probability one the are times when the origin is visited only a finite number of times while other points are visited infinitely often.
\end{abstract}

Key words: Random Walks; Dynamical Random Walks, Dynamical Sensitivity.

AMS 2000 Subject Classification: Primary 60G50 ; 82C41.

Submitted to EJP on October 9, 2006, final version accepted October 20, 2008.

\footnotetext{
*Weizmann Institute, Rehovot, 76100, Israel. Email: gideon.amir@weizmann.ac.il

${ }^{\dagger}$ Funded in part by an MSRI, DMS grant \#0501102 and the University of Washington Royalty Research Fund
} 


\section{Introduction}

We consider a dynamical simple random walk on $\mathbb{Z}^{2}$. Associated with each $n$ is a Poisson clock. When the clock rings the $n$th move of the random walk is replaced by an independent random variable with the same distribution. Thus for any fixed $t$ the distribution of the walks at time $t$ is that of simple random walk on $\mathbb{Z}^{2}$ and is almost surely recurrent.

More formally let $\left\{Y_{n}^{m}\right\}_{m, n \in \mathbb{N}}$ be uniformly distributed i.i.d. random variables chosen from the set $\{(0,1),(0,-1),(1,0),(-1,0)\}$.

For each $n$, let $\left\{\tau_{n}^{(m)}\right\}_{m \geq 0}$ be the arrival times of a Poisson process of rate 1 , with the processes for different $n$ independent of one another and $\tau_{n}^{(0)}=0$ for each $n$.

Define

$$
X_{n}(t)=Y_{n}^{m}
$$

for all $t \in\left[\tau_{n}^{(m)}, \tau_{n}^{(m+1)}\right)$. Let

$$
S_{n}(t)=\sum_{i=1}^{n} X_{i}(t)
$$

Thus for each $t$ the random variables $\left\{X_{n}(t)\right\}_{n \in \mathbb{N}}$ are i.i.d.

The concept of dynamical random walk (and related dynamical models) was introduced by Benjamini, Häggström, Peres and Steif in [2], where they showed various properties of the simple random walk to hold for all times almost surely (such properties are called "dynamically stable"), while for other properties there a.s. exist exceptional times for which they do not hold (and are then called "dynamically sensitive" properties). In particular, they showed that transience of simple random walk is dynamically sensitive in 3 and 4 dimensions, while in dimension 5 or higher it is dynamically stable. For more work on dynamical models see also [8] and [4].

In [5] Hoffman showed that recurrence of simple random walk in two dimensions is dynamically sensitive, and that the Hausdorff dimension of the set of exceptional times is a.s. 1. Furthermore it was showed that there exist exceptional times where the walk drifts to infinity with rate close to $\sqrt{n}$.

The following problem was raised by Jeff Steif and Noam Berger during their stay at MSRI: When the dynamical random walk on $\mathbb{Z}^{2}$ is in an exceptional time, returning to the origin only a finite number of times, is it always because it "escapes to infinity", or are there times where the disc of radius 1 is hit infinitely often, but the origin is hit only a finite number of times.

In this paper we prove that such exceptional times exist, and furthermore that the Hausdorff dimension of the sets of these exceptional times is 1 . The techniques used are a refinement of the techniques used in [5] to show the existence of exceptional times where the walk is transient.

The proof is divided into two main parts. First, we define a sequence of events $S E_{k}(t)$, dependent on $\left\{S_{n}(t)\right\}_{n \in \mathbb{N}}$ such that $\bigcap S E_{k}(t)$ implies we are in an exceptional time of the desired form, and show that the correlation between these events in two different times $t_{1}, t_{2}$ is sufficiently small (Theorem 2). Then we use this small correlation event, in a manner quite similar to [5] to show the existence of a dimension 1 set of exceptional times. 


\section{Definitions, Notation and Main Results}

We will follow the notation of [5] as much as possible. Let $\mathbb{D}$ denote the discrete circle of radius 1 :

$$
\mathbb{D}=\{(1,0),(-1,0),(0,1),(0,-1)\} .
$$

The set of special exceptional times of the process, $S^{\text {exc }}$, is the set of all times for which the walk visits $\mathbb{D}$ infinitely often but visits $(0,0)$ only fintely often. More explicitly we define

$$
S^{\mathrm{exc}}=\left\{t:\left|\left\{n: S_{n}(t) \in \mathbb{D}\right\}\right|=\infty \text { and }\left|\left\{n: S_{n}(t)=0\right\}\right|<\infty\right\} .
$$

The main result of this paper is:

Theorem 1. $\mathbf{P}\left(S^{\text {exc }} \neq \emptyset\right)=1$. Furthermore the Hausdorff dimension of $S^{\text {exc }}$ is 1 a.s.

We will need the following notation. Define a series of times

$$
s_{k}=k^{10} 2^{2 k^{2}}
$$

We will divide the walk into segments between two consecutive $s_{i}$ :

$$
M_{j}=\left\{i \in \mathbb{N}: s_{j-1} \leq i<s_{j}\right\},
$$

And define a super segment to be a union of segments

$$
W_{k}=\bigcup_{2^{k} \leq j<2^{k+1}} M_{j}=\left\{i \in \mathbb{N}: s_{2^{k}-1} \leq i<s_{2^{k+1}}\right\} .
$$

Define a series of annuli

$$
A_{k}=\left\{x \in \mathbb{Z}^{2}: 2^{k^{2}} \leq|x| \leq k^{10} 2^{2 k^{2}}\right\}
$$

where $|x|$ denotes the standard Euclidean norm $|x|=\sqrt{x_{1}^{2}+x_{2}^{2}}$.

And define event $G_{k}$ by

$$
G_{k}(t)=\left\{S_{s_{k}}(t) \in A_{k}\right\}
$$

Choosing these annuli to be of the right magnitude will prove to be one of the most useful tools at our disposal. We will show that a random walk will typically satisfy $G_{k}$ (see Lemmas 6 and 7). On the other hand, the location of the walk inside the annuli $A_{j}$ in the beginning of the segment $M_{j}$ will have only a small influence of the probabilities of the events we will investigate (see Lemmas 8 and 10). Together this provides "almost independence" of events in different segments.

We will define three events concerned with hitting the disc on the dynamical random walk. We define the event $R_{j}(t)$, to be the event that the walk (at time $t$ ) hits $\mathbb{D}$ at some step in in the segment $M_{j}$, i.e.

$$
R_{j}(t):=\left\{\exists m \in M_{j} \text { such that } S_{m}(t) \in \mathbb{D}\right\}
$$


(Note that in [5], this denoted the event of hitting the origin, and not $\mathbb{D}$, but this difference does not alter any of the calculations.)

We denote by $S R_{j}(t)$ the event that the walk (at time $t$ ) hits $\mathbb{D}$ at some step in the segment $M_{j}$, but does not hit the origin at any step of $M_{j}$ :

$$
S R_{j}(t):=\left\{\left(\exists n \in M_{j} \text { such that } S_{n}(t) \in \mathbb{D}\right) \cap\left(\forall m \in M_{j} S_{m}(t) \neq(0,0)\right)\right\} .
$$

It is easy to see that $S R_{j}(t) \subset R_{j}(t)$.

We define the third event, $S E_{k}(t)$, by

$$
\left.S E_{k}(t)=\left\{\bigcup_{j=2^{k}}^{2^{k+1}-1}\left(S R_{j}(t) \cap\left(\bigcap_{i=2^{k}, i \neq j}^{2^{k+1}}\left(R_{i}(t)\right)^{c}\right)\right)\right\} \bigcap \bigcap_{i=2^{k}}^{2^{k+1}} G_{i}(t)\right\} .
$$

This is the event that the walk never hits the origin in the super segment $W_{k}$, and there is exactly one segment $M_{j}, 2^{k} \leq j<2^{k}$, where the walk hits $\mathbb{D}$. Additionally this event requires the walk ends each segment $M_{i}$ in the annulus $A_{i}$.

It is easy to see that if the event $\bigcap_{k \geq M} S E_{k}(t)$ occurs for some integer $M$ then $t \in S^{\text {exc }}$.

Given $2^{k} \leq j<2^{k+1}$ we write

$$
S E_{k}^{j}(0)=S E_{k}(0) \cap S R_{j}(0) .
$$

For any random walk event $E$ we write $E(0, t)$ for $E(0) \cap E(t)$. Thus for example, we have

$$
S R_{j}(0, t)=S R_{j}(0) \cap S R_{j}(t) .
$$

For $j_{1}, j_{2} \in\left[2^{k}, 2^{k+1}\right)$ we write

$$
S E_{k}^{j_{1}, j_{2}}(0, t)=S E_{k}(0, t) \cap S R_{j_{1}}(0) \cap S R_{j_{2}}(t) .
$$

We will use the following notation for conditional probabilities. Let

$$
\mathbf{P}^{x, k-1}(*)=\mathbf{P}\left(* \mid S_{s_{k-1}}(0)=x\right)
$$

and

$$
\mathbf{P}^{x, y, k-1}(*)=\mathbf{P}\left(* \mid S_{s_{k-1}}(0)=x \text { and } S_{s_{k-1}}(t)=y\right) .
$$

To prove Theorem 1 we will first prove the following theorem.

Theorem 2. There is a function $f(t)$ such that for any $M$,

$$
\frac{\mathbf{P}\left(\bigcap_{1 \leq k \leq M} S E_{k}(0, t)\right)}{\left(\mathbf{P} \bigcap_{1 \leq k \leq M}\left(S E_{k}(0)\right)\right)^{2}} \leq f(t)
$$

and $\int_{0}^{1} f(t)<\infty$. 
The first statement in Theorem 1 follows from Theorem 2 and the second moment method. The second statement in Theorem 1 will follow from bounds that we will obtain on the growth of $f(t)$ near zero and by Lemma 5.1 of [11].

Since we will be showing that some events have low correlation between times 0 and $t$, some of the bounds we use to prove Theorem 2 will only hold when $k$ is sufficiently large compared to $\frac{1}{t}$. Therefore, for $t \in(0,1)$, we denote by $K(t)$ the smallest integer greater than $|\log t|+1$, and by $K^{\prime}(t)$ the smallest integer greater than $\log (|\log t|+1)+1$. (Here and everywhere we use $\log (n)=\log _{2}(n)$ ). We end this section by stating that we use $C$ as a generic constant whose value may increase from line to line and lemma to lemma. In many of the proofs in the next section we use bounds that only hold for sufficiently large $k$ (or $j$ ). This causes no problem since it will be clear that we can always choose $C$ such that the lemma is true for all $k$.

\section{Proof of Theorem 2}

This section is divided into four parts. In the first, we introduce some bounds on the behavior of two dimensional simple random walk. These are quite standard, and are brought here without proof. In the second part we quote, for the sake of completeness, lemmas from [5] which we will use later on. In the third part, which constitutes the bulk of this paper, we prove estimates on $\mathbf{P}\left(S E_{k}(0) \mid S E_{k-1}(0)\right)$ and $\mathbf{P}\left(S E_{k}(0, t) \mid S E_{k-1}(0, t)\right)$. In the fourth part we use the these bounds to prove Theorem 2 .

\subsection{Two Dimensional Simple Random Walk Lemmas}

The main tool that we use are bounds on the probability that simple random walk started at $x$ returns to the origin before exiting the ball of radius $n$ with center at the origin. For general $x$, this probability is calculated in Proposition 1.6.7 on page 40 of [7] in a stronger form than given here. The estimate for $|x|=1$ comes directly from estimates on the $2-d$ harmonic potential (Spitzer 76, P12.3 page 124 or [6]).

Let $\eta$ be the smallest $m>0$ such that $S_{m}(0)=0$ or $\left|S_{m}(0)\right| \geq n$.

Lemma 3. 1. There exists $C>0$ such that for all $x$ with $0<|x|<n$

$$
\frac{\log (n)-\log |x|-C}{\log (n)} \leq \mathbf{P}\left(S_{\eta}(0)=\mathbf{0} \mid S_{0}(0)=x\right) \leq \frac{\log (n)-\log |x|+C}{\log (n)} .
$$

2. For $|x|=1$ we have the following stronger bound:

$$
P\left(\left|S_{\eta}(0)\right| \geq n \mid S_{0}(0)=x\right)=\frac{\pi}{2 \log n+C}+O\left(n^{-1}\right) .
$$

We will also use the following standard bounds.

Lemma 4. There exists $C>0$ such that for all $x \in \mathbb{Z}^{2}, n \in \mathbb{N}$ and $m<\sqrt{n}$

$$
\mathbf{P}\left(\exists n^{\prime}<n: S_{n^{\prime}}(0)>m \sqrt{n}\right) \leq \frac{C}{m^{2}}
$$


and

$$
\mathbf{P}\left(\left|S_{n}(0)-x\right|<\frac{\sqrt{n}}{m}\right) \leq \frac{C}{m^{2}} .
$$

Lemma 4 is most frequently applied in the form of the following corollary:

Corollary 5. There exists $C>0$, such that for any $n_{0}, N, M>0$, if $\left|S_{n_{0}}(0)\right| \geq M$ then

$$
\mathbf{P}\left(\exists n \text { such that } n_{0}<n<n_{0}+N \text { and }\left|S_{n}(0)\right| \leq 1\right) \leq \frac{C N}{M^{2}} \text {. }
$$

\subsection{Some Useful Lemmas From [5]}

First we list some lemmas from [5] that we will use in the proof. (In [5] $R_{j}(0)$ is defined as

$$
R_{j}(0)=\left\{\exists m \in M_{j} \text { such that } S_{m}(0)=0\right\} .
$$

It is easy to see all lemmas in [5] hold with our slightly expanded definition.)

Lemma 6 shows that if the walk is in the annulus $A_{j-1}$ at step $s_{j-1}$, then with high probability the walk will be in the annulus $A_{j}$ at step $s_{j}$. This will allow us to condition our estimates of various events on the event that $S_{s_{j}} \in A_{j}$.

Lemma 6. For any $j$ and $x \in A_{j-1}$

$$
\mathbf{P}^{x, j-1}\left(\left(G_{j}(0)\right)^{C}\right) \leq \frac{C}{j^{10}}
$$

We will usually use the following corollary of Lemma 6, which is just an application of Lemma 6 to all segments inside a super-segment, and follows directly by applying the union bound:

Corollary 7. For any $k>0$ and $x \in A_{2^{k}-1}$

$$
\mathbf{P}^{x, 2^{k}-1}\left(\left(\bigcap_{2^{k} \leq l<2^{k+1}} G_{l}(0)\right)^{c}\right) \leq \frac{C}{2^{9 k}} .
$$

Lemma 8 shows that we have a good estimate of the probability of hitting $\mathbb{D}$ during the segment $M_{k}$, given the walk starts the segment inside the annulus $A_{k-1}$.

Lemma 8. There exists $C$ such that for any $k$ and any $x \in A_{k-1}$

$$
\frac{2}{k}-\frac{C \log k}{k^{2}} \leq \mathbf{P}^{x, k-1}\left(R_{k}(0)\right) \leq \frac{2}{k}+\frac{C \log k}{k^{2}}
$$

Lemma 9 tells us that resampling a small percentage of the moves is enough to get a very low correlation between hitting $\mathbb{D}$ before and after the re-randomization. 
Lemma 9. There exists $C$ such that for all $k, n \geq s_{k-1}+s_{k} / 2^{10 k}$, for all $I \subset\{1, \ldots, n\}$ with $|I| \geq s_{k} / 2^{10 k}$ and for all $\left\{X_{i}(t)\right\}_{i \in\{1, \ldots, n\} \backslash I}$

$$
\mathbf{P}\left(\exists j \in\left\{n, \ldots, s_{k}\right\} \text { such that } S_{j}(t) \in \mathbb{D} \mid\left\{x_{i}(t)\right\}_{i \in\{1, \ldots, n\} \backslash I}\right) \leq \frac{C}{k} .
$$

(Where we interpret the set $\left\{n, \ldots, s_{k}\right\}$ as the empty set for $n>s_{k}$ ).

The last lemma we quote says there is a low correlation between hitting $\mathbb{D}$ (during some segment $M_{k}$ ) at different times.

Lemma 10. There exists $C$ such that for any $t$, any $k>K(t)$ and any $x, y \in A_{k-1}$

$$
\mathbf{P}^{x, y, k-1}\left(R_{k}(0, t)\right) \leq \frac{C}{k^{2}} \text {. }
$$

\subsection{Estimating $\mathbf{P}\left(S E_{k}(0)\right)$ and $\mathbf{P}\left(S E_{k}(0, t)\right)$}

We start by giving estimates for the event $S R_{j}(t)$.

Lemma 11. There exists $C>0$, such that for any $j$ and any $x \in A_{j-1}$,

$$
\frac{\pi}{j^{3}}-\frac{C \log j}{j^{4}} \leq \mathbf{P}^{x, j-1}\left(S R_{j}(0)\right) \leq \frac{\pi}{j^{3}}+\frac{C \log j}{j^{4}} .
$$

Proof. Let $h$ be the first step in the segment $M_{j}$ such that $S_{h}(0) \in \mathbb{D}$, letting $h=\infty$ if no such step exists. By Lemma 8 ,

$$
\frac{2}{j}-\frac{C \log j}{j^{2}} \leq \mathbf{P}^{x, j-1}(h<\infty) \leq \frac{2}{j}+\frac{C \log j}{j^{2}} .
$$

Let $B_{i}$ denote the event that the walk does not hit $\mathbf{0}$ between step $i$ and $s_{j}$. Then

$$
\begin{aligned}
\mathbf{P}^{x, j-1} & \left(S R_{j}(0) \cap G_{j}(0)\right) \\
& =\sum_{s_{j-1} \leq i<s_{j}} \mathbf{P}^{x, j-1}(h=i) \mathbf{P}\left(B_{i} \cap G_{j}(0) \mid h=i\right) .
\end{aligned}
$$

For any $s_{j-1} \leq i<s_{j}, B_{i} \cap G_{j}(0)$ is included in the event that after step $i$, the walk reaches $A_{j}$ before reaching 0 . Therefore by the second statement in Lemma 3 there exists $C$ such that for all $i$

$$
\mathbf{P}\left(B_{i}(0) \cap G_{j}(0) \mid h=i\right) \leq \frac{\pi}{2 j^{2}}+\frac{C}{j^{4}} .
$$

Putting (5) and (3) into (4) we get 


$$
\begin{aligned}
\mathbf{P}^{x, j-1}\left(S R_{j}(0)\right. & \left.\cap G_{j}(0)\right) \\
& =\sum_{i} \mathbf{P}^{x, j-1}(h=i) \cdot \mathbf{P}\left(B_{i} \cap G_{j}(0) \mid h=i\right) \\
& \leq \sum_{i} \mathbf{P}^{x, j-1}(h=i) \cdot \sup _{i}\left(\mathbf{P}\left(B_{i} \cap G_{j}(0) \mid h=i\right)\right) \\
& \leq \mathbf{P}^{x, j-1}(h<\infty) \cdot\left(\frac{\pi}{2 j^{2}}+\frac{C}{j^{4}}\right) \\
& \leq\left(\frac{2}{j}+\frac{C \log j}{j^{2}}\right) \cdot\left(\frac{\pi}{2 j^{2}}+\frac{C}{j^{4}}\right) \\
& \leq \frac{\pi}{j^{3}}+\frac{C \log j}{j^{4}} .
\end{aligned}
$$

By Lemma 6 we have that for all $x \in A_{j-1}$

$$
\mathbf{P}^{x, j-1}\left(G_{j}(0)^{c}\right) \leq \frac{C}{j^{10}} .
$$

Thus using (6) and (7) together with conditional probabilities with respect to $G_{j}(0)$, we get

$$
\begin{aligned}
\mathbf{P}^{x, j-1}\left(S R_{j}(0)\right) & \leq \mathbf{P}^{x, j-1}\left(S R_{j}(0) \cap G_{j}(0)\right)+\mathbf{P}^{x, j-1}\left(G_{j}(0)^{c}\right) \\
& \leq \frac{\pi}{j^{3}}+\frac{C \log j}{j^{4}}+\frac{C}{j^{10}} \\
& \leq \frac{\pi}{j^{3}}+\frac{C \log j}{j^{4}} .
\end{aligned}
$$

which establishes the upper bound.

To get the lower bound, note that conditioned on the event $G_{j}(0)$, if after step $i$ the walk reaches $A_{j}$ before reaching $(0,0)$, and then does not hit $(0,0)$ in the next $s_{j}$ steps, then $B_{i}$ occurs.

Letting $E_{1}$ denote the event that after step $i$ we reach $A_{j}$ before reaching $(0,0)$, and letting $E_{2}$ denote the event that a simple random walk, starting at $A_{j}$, does not hit $(0,0)$ in the next $s_{j}$ steps. We get, using the Markov property, that

$$
\mathbf{P}^{x, j-1}\left(B_{i} \mid G_{j}(0) \cap\{h=i\}\right) \geq \mathbf{P}\left(E_{1} \mid h=i\right) \mathbf{P}\left(E_{2}\right) .
$$

By Lemma 3 we have

$$
\mathbf{P}\left(E_{1} \mid h=i\right) \geq\left(\frac{\pi}{2 j^{2}}-\frac{C}{j^{4}}\right) .
$$

And by the Markov property and stationarity, since a walk starting at $A_{j}$ must hit $A_{j-1}$ before reaching 0 , we can use Lemma 8 to bound

$$
\mathbf{P}\left(E_{2}\right) \geq\left(1-\frac{2}{j}-\frac{C \log j}{j^{2}}\right) .
$$


Combining (9) and (10) into (8) we get

$$
\begin{aligned}
\mathbf{P}^{x, j-1}\left(B_{i} \mid G_{j}(0) \cap\{h=i\}\right) & \geq\left(\frac{\pi}{2 j^{2}}-\frac{C}{j^{4}}\right)\left(1-\frac{2}{j}-\frac{C \log j}{j^{2}}\right) \\
& \geq \frac{\pi}{2 j^{2}}-\frac{C}{j^{3}} .
\end{aligned}
$$

Using (11) for all $j$ and $x \in A_{j-1}$

$$
\begin{aligned}
\mathbf{P}^{x, j-1}\left(S R_{j}(0) \mid G_{j}(0)\right) & =\sum_{i} \mathbf{P}^{x, j-1}(h=i) \mathbf{P}\left(B_{i} \mid G_{j}(0) \cap\{h=i\}\right) \\
& \geq \mathbf{P}^{x, j-1}(h<\infty) \inf _{i} \mathbf{P}\left(B_{i} \mid G_{j}(0) \cap\{h=i\}\right) \\
& \geq\left(\frac{2}{j}-\frac{C \log j}{j^{2}}\right) \cdot\left(\frac{\pi}{2 j^{2}}-\frac{C}{j^{3}}\right) \\
& \geq \frac{\pi}{j^{3}}-\frac{C \log j}{j^{4}} .
\end{aligned}
$$

Thus for all $j$ and $x \in A_{j-1}$, conditioning on $G_{j}(0)$ and using Lemma 6 and (12) implies that

$$
\begin{aligned}
\mathbf{P}^{x, j-1}\left(S R_{j}(0)\right) & \geq \mathbf{P}^{x, j-1}\left(S R_{j}(0) \mid G_{j}(0)\right)-\mathbf{P}^{x, j-1}\left(G_{j}(0)^{c}\right) \\
& \geq \frac{\pi}{j^{3}}-\frac{C \log j}{j^{4}}-\frac{C}{j^{10}} \\
& \geq \frac{\pi}{j^{3}}-\frac{C \log j}{j^{4}} .
\end{aligned}
$$

The next two lemmas give a lower bound on $\mathbf{P}\left(S E_{k}(0)\right)$. We define

$$
\beta_{k}:=\prod_{2^{k} \leq j<2^{k+1}}\left(1-\frac{2}{j}\right) .
$$

This is an approximation of the probability of never hitting $\mathbb{D}$ during the super segment $W_{k}$. Note that there exists $c>0$ such that $c<\beta_{k}<1$ for all $k>1$.

Lemma 12. For any $k$ and any $2^{k} \leq j<2^{k+1}$, and any $x \in A_{2^{k}-1}$

$$
\mathbf{P}^{x, 2^{k}-1}\left(S E_{k}^{j}(0)\right) \geq \beta_{k} \frac{\pi}{j^{3}}-\frac{C \log j}{j^{4}} .
$$

Proof. By the Markov property, for any $x \in A_{2^{k}-1}$ 


$$
\begin{aligned}
\mathbf{P}^{x, 2^{k}-1} & \left(S E_{k}^{j}(0)\right) \\
\geq & \min _{x \in A_{j-1}} \mathbf{P}^{x, j-1}\left(S R_{j}(0) \cap G_{j}(0)\right) . \\
& \cdot \prod_{2^{k} \leq l<2^{k+1}, l \neq j}\left(\min _{x \in A_{l-1}} \mathbf{P}^{x, l-1}\left(\left(R_{l}(0) \cap G_{l}(0)\right)^{c}\right)\right. \\
\geq & \min _{x \in A_{j-1}} \mathbf{P}^{x, j-1}\left(S R_{j}(0)\right) \prod_{2^{k} \leq l<2^{k+1}, l \neq j}\left(\min _{x \in A_{l-1}} \mathbf{P}^{x, l-1}\left(R_{l}(0)\right)^{c}\right) \\
& -\sum_{2^{k} \leq l<2^{k+1}} \mathbf{P}^{x, l-1}\left(\left(G_{l}\right)^{c}\right)
\end{aligned}
$$

By Lemma 11

$$
\min _{x \in A_{j-1}} \mathbf{P}^{x, j-1}\left(S R_{j}(0)\right) \geq \frac{\pi}{j^{3}}-\frac{C \log j}{j^{4}} .
$$

By Lemma 8

$$
\min _{x \in A_{l-1}} \mathbf{P}^{x, l-1}\left(\left(R_{l}(0)\right)^{c}\right) \geq\left(1-\frac{2}{l}\right)-\frac{C \log l}{l^{2}} .
$$

Multiplying over all $l$ such that $2^{k} \leq l<2^{k+1}$ and $l \neq j$, we get

$$
\begin{aligned}
\prod_{2^{k} \leq l<2^{k+1}, l \neq j} & \left(\min _{x \in A_{l-1}} \mathbf{P}^{x, l-1}\left(\left(R_{l}(0)\right)^{c}\right)\right) \\
& \geq \prod_{2^{k} \leq l<2^{k+1}, l \neq j}\left(1-\frac{2}{l}-\frac{C \log l}{l^{2}}\right) \\
& \geq \prod_{2^{k} \leq l<2^{k+1}, l \neq j}\left(1-\frac{2}{l}\right) \\
& -\prod_{2^{k} \leq l<2^{k+1}, l \neq j}\left(\frac{C \log l}{l^{2}} \prod_{2^{k} \leq m<2^{k+1}, m \neq l, j}\left(1-\frac{2}{m}\right)\right) \\
& \geq \prod_{2^{k} \leq l<2^{k+1}}\left(1-\frac{2}{l}\right)-\sum_{2^{k} \leq l<2^{k+1}} \frac{C \log l}{l^{2}} \prod_{2^{k} \leq l<2^{k+1}}\left(1-\frac{2}{l}\right)-2^{k} \frac{C \log 2^{k}}{2^{2 k}} \prod_{2^{k} \leq l<2^{k+1}}\left(1-\frac{2}{l}\right) \\
\geq & \beta_{k}-\frac{\beta_{k} C \log 2^{k}}{2^{k}} \\
\geq & \beta_{k}-\frac{C \log j}{j} .
\end{aligned}
$$

Last, by Lemma 6

$$
\sum_{2^{k} \leq l<2^{k+1}} \mathbf{P}^{x, l-1}\left(\left(G_{l}\right)^{c}\right) \leq 2^{k} \frac{C}{2^{10 k}} \leq \frac{C}{2^{9 k}} .
$$


Putting (15),(16) and (17) into (14) we get

$$
\begin{aligned}
& \mathbf{P}^{x, 2^{k}-1}\left(S E_{k}^{j}(0)\right) \\
& \geq \min _{x \in A_{j-1}} \mathbf{P}^{x, j-1}\left(S R_{j}(0)\right) \prod_{2^{k} \leq l<2^{k+1}, l \neq j}\left(\min _{x \in A_{l-1}} \mathbf{P}^{x, l-1}\left(R_{l}(0)\right)^{c}\right) \\
& \quad-\sum_{2^{k} \leq l<2^{k+1}} \mathbf{P}^{x, l-1}\left(\left(G_{l}\right)^{c}\right) \\
& \geq\left(\frac{\pi}{j^{3}}-\frac{C \log j}{j^{4}}\right)\left(\beta_{k}-\frac{C \log j}{j}\right)-\frac{C}{2^{9 k}} \\
& \geq \beta_{k} \frac{\pi}{j^{3}}-\frac{C \log j}{j^{4}} .
\end{aligned}
$$

Lemma 13.

$$
\mathbf{P}\left(S E_{k}(0) \mid S E_{k-1}(0)\right) \geq \pi \beta_{k}\left(\sum_{2^{k} \leq j<2^{k+1}} \frac{1}{j^{3}}\right)-\frac{C k}{2^{3 k}} .
$$

Proof. $S E_{k-1}(0) \subseteq G_{2^{k}-1}(0)$, and therefore by the Markov property of simple random walk, we have

$$
\mathbf{P}\left(S E_{k}(0) \mid S E_{k-1}(0)\right) \geq \min _{x \in A^{2^{k}-1}} \mathbf{P}^{x, 2^{k}-1}\left(S E_{k}(0)\right) .
$$

By the definition of $S E_{k}^{j}$, the events $S E_{k}^{j_{1}}$ and $S E_{k}^{j_{2}}$ are disjoint for $j_{1} \neq j_{2}$, so

$$
\begin{aligned}
\min _{x \in A^{2^{k}-1}} \mathbf{P}^{x, 2^{k}-1}\left(S E_{k}(0)\right) & =\min _{x \in A^{2^{k}-1}} \sum_{2^{k} \leq j<2^{k+1}} \mathbf{P}^{x, 2^{k}-1}\left(S E_{k}^{j}(0)\right) \\
& \geq \sum_{2^{k} \leq j<2^{k+1}}\left(\min _{x \in A^{2^{k}-1}} \mathbf{P}^{x, 2^{k}-1}\left(S E_{k}^{j}(0)\right)\right) \\
& \geq \sum_{2^{k} \leq j<2^{k+1}}\left(\beta_{k} \frac{\pi}{j^{3}}-\frac{C \log j}{j^{4}}\right) \\
& \geq \pi \beta_{k}\left(\sum_{2^{k} \leq j<2^{k+1}} \frac{1}{j^{3}}\right)-\frac{C k}{2^{3 k}} .
\end{aligned}
$$

The inequality in (18) follows from Lemma 12.

Next we will work to bound from above the probability of $S E_{k}(0, t)$.

First, we deal with the case that the walk hits $\mathbb{D}$ in different sub-segments of $W_{k}$ in times 0 and $t$. 
Lemma 14. There exists a constant $C>0$ such that for any $k>K^{\prime}(t)$ and any $j_{1} \neq j_{2}, 2^{k} \leq j_{1}, j_{2}<$ $2^{k+1}$, we have

$$
\max _{x, y \in A_{2^{k}-1}} \mathbf{P}^{x, y, 2^{k}-1}\left(S E_{k}^{j_{1}, j_{2}}(0, t)\right) \leq \beta_{k}^{2} \frac{\pi}{j_{1}^{3}} \frac{\pi}{j_{2}^{3}}+\frac{C k}{2^{7 k}} .
$$

Proof. Recall that

$$
\begin{aligned}
& S E_{k}^{j_{1}, j_{2}}(0, t)= \\
& \quad\left\{S R_{j_{1}}(0) \cap\left(R_{j_{1}}(t)\right)^{c}\right\} \cap\left\{\left(R_{j_{2}}(0)\right)^{c} \cap S R_{j_{2}}(t)\right\} \cap \\
& \quad\left\{\bigcap_{2^{k} \leq l<2^{k+1}, l \neq j_{1}, j_{2}}\left(\left(R_{l}(0)\right)^{c} \cap\left(R_{l}(t)\right)^{c}\right)\right\} \cap\left\{\bigcap_{2^{k} \leq l<2^{k+1}}\left(G_{l}(0, t)\right)^{c}\right\} .
\end{aligned}
$$

So by the Markov property,

$$
\begin{aligned}
\max _{x, y \in A_{2^{k}-1}} \mathbf{P}^{x, y, 2^{k}-1}\left(S E_{k}^{j_{1}, j_{2}}(0, t)\right) \\
\leq \max _{x, y \in A_{j_{1}-1}} \mathbf{P}^{x, y, j_{1}-1}\left(S R_{j_{1}}(0) \cap\left(R_{j_{1}}(t)\right)^{c}\right) \\
\quad \cdot \max _{x, y \in A_{j_{2}-1}} \mathbf{P}^{x, y, j_{2}-1}\left(\left(R_{j_{2}}(0)\right)^{c} \cap S R_{j_{2}}(t)\right) \\
\quad \cdot \prod_{2^{k} \leq l<2^{k+1}, l \neq j_{1}, j_{2}} \max _{x, y \in A_{l-1}} \mathbf{P}^{x, y, l-1}\left(\left(R_{l}(0)\right)^{c} \cap\left(R_{l}(t)\right)^{c}\right) \\
\quad+\max _{x, y \in A_{l-1}} \mathbf{P}^{x, y, 2^{k}-1}\left(\left(\bigcap_{2^{k} \leq l<2^{k+1}} G_{l}(0, t)\right)^{c}\right) .
\end{aligned}
$$

Now we will estimate each of the four terms in (19).

$$
\begin{aligned}
\max _{x, y \in A_{j_{1}-1}} \mathbf{P}^{x, y, j_{1}-1}\left(S R_{j_{1}}(0) \cap\left(R_{j_{1}}(t)\right)^{c}\right) & \leq \max _{x \in A_{j_{1}-1}} \mathbf{P}^{x, j_{1}-1}\left(S R_{j_{1}}(0)\right) \\
& \leq \frac{\pi}{j_{1}^{3}}+\frac{C \log j_{1}}{j_{1}^{4}}
\end{aligned}
$$

The last inequality holding by Lemma 11 .

Similarly, by symmetry between times 0 and $t$,

$$
\max _{x, y \in A_{j_{2}-1}} \mathbf{P}^{x, y, j_{2}-1}\left(\left(R_{j_{2}}(0)\right)^{c} \cap S R_{j_{2}}(t)\right) \leq \frac{\pi}{j_{2}^{3}}+\frac{C \log j_{2}}{j_{2}^{4}} .
$$

To estimate the third term in (19) we use Lemma 10 to estimate each term in the product. Lemma 10 says that there exists $C>0$ such that for any $l$ and any $x, y \in A_{l-1}$

$$
\mathbf{P}^{x, y, l-1}\left(R_{l}(0, t)\right) \leq \frac{C}{l^{2}} .
$$


And the lower bound from Lemma 8:

$$
\mathbf{P}^{x, l-1}\left(R_{l}(0)\right) \geq \frac{2}{l}-\frac{C \log l}{l^{2}} .
$$

We then get that

$$
\begin{aligned}
& \max _{x, y \in A_{l-1}} \mathbf{P}^{x, y, l-1}\left(\left(R_{l}(0)\right)^{c} \cap\left(R_{l}(t)\right)^{c}\right) \\
& \leq 1-\min _{x, y \in A_{l-1}} \mathbf{P}^{x, y, l-1}\left(R_{l}(0)\right) \\
& \quad-\min _{x, y \in A_{l-1}} \mathbf{P}^{x, y, l-1}\left(R_{l}(t)\right)+\max _{x, y, l-1} \mathbf{P}^{x, y, l-1}\left(R_{l}(0, t)\right) \\
& \leq \quad 1-2 \min _{x \in A_{l-1}} \mathbf{P}^{x, l-1}\left(R_{l}(0)\right)+\max _{x, y, l-1} \mathbf{P}^{x, y, l-1}\left(R_{l}(0, t)\right) \\
& \leq 1-\frac{4}{l}+\frac{C \log l}{l^{2}} .
\end{aligned}
$$

Which yields

$$
\begin{aligned}
\prod_{2^{k} \leq l<2^{k+1}, l \neq j_{1}, j_{2}} & \max _{x, y, l-1} \mathbf{P}^{x, y, l-1}\left(\left(R_{l}(0)\right)^{c} \cap\left(R_{l}(t)\right)^{c}\right) \\
\leq & \prod_{2^{k} \leq l<2^{k+1}, l \neq j_{1}, j_{2}}\left(1-\frac{4}{l}+\frac{C \log l}{l^{2}}\right) \\
\leq & \prod_{2^{k} \leq l<2^{k+1}, l \neq j_{1}, j_{2}}\left(1-\frac{4}{l}+\frac{4}{l^{2}}+\frac{C \log l}{l^{2}}\right) \\
\leq & \prod_{2^{k} \leq l<2^{k+1}, l \neq j_{1}, j_{2}}\left(1-\frac{4}{l}+\frac{4}{l^{2}}\right) \\
& +\sum_{2^{k} \leq l<2^{k+1}, l \neq j_{1}, j_{2}}\left(\frac{C \log l}{l^{2}} \sum_{2^{k} \leq m<2^{k+1}, m \neq l, j_{1}, j_{2}}\left(1-\frac{4}{m}+\frac{4}{m^{2}}\right)\right) \\
\leq & \frac{\beta_{k}^{2}}{\left(1-\frac{2}{j_{1}}\right)^{2}\left(1-\frac{2}{j_{2}}\right)^{2}}+\sum_{2^{k} \leq l<2^{k+1}, l \neq j_{1}, j_{2}} \frac{C \log l}{l^{2}} \\
\leq & \beta_{k}^{2}+\frac{C k}{2^{k}} .
\end{aligned}
$$

For the fourth factor in (19), Corollary 7 gives

$$
\begin{aligned}
\max _{x, y \in A_{l-1}} \mathbf{P}^{x, y, 2^{k}-1} & \left(\bigcap_{2^{k} \leq l<2^{k+1}} G_{l}(0, t)\right)^{c} \\
& \leq \mathbf{P}^{x, 2^{k}-1}\left(\bigcap_{2^{k} \leq l<2^{k+1}} G_{l}(0)\right)^{c}+\mathbf{P}^{y, 2^{k}-1}\left(\bigcap_{2^{k} \leq l<2^{k+1}} G_{l}(0)\right)^{c} \\
& \leq \frac{C}{2^{9 k}}
\end{aligned}
$$


Combining (20), (21), (22) and (23) into (19) we get

$$
\begin{aligned}
\max _{x, y \in A_{2^{k}-1}} \mathbf{P}^{x, y, 2^{k}-1}\left(S E_{k}^{j_{1}, j_{2}}(0, t)\right) \\
\leq\left(\frac{\pi}{j_{1}^{3}}+\frac{C \log j_{1}}{j_{1}^{4}}\right) \cdot\left(\frac{\pi}{j_{2}^{3}}+\frac{C \log j_{2}}{j_{2}^{4}}\right) \cdot\left(\beta_{k}^{2}+\frac{C k}{2^{k}}\right)+\frac{C}{2^{9 k}} \\
\leq\left(\frac{\pi}{j_{1}^{3}}+\frac{C k}{2^{4 k}}\right) \cdot\left(\frac{\pi}{j_{2}^{3}}+\frac{C k}{2^{4 k}}\right) \cdot\left(\beta_{k}^{2}+\frac{C k}{2^{k}}\right)+\frac{C}{2^{9 k}} \\
\leq \beta_{k}^{2} \frac{\pi}{j_{1}^{3}} \frac{\pi}{j_{2}^{3}}+\frac{C k}{2^{7 k}} .
\end{aligned}
$$

Next, we deal with the case in which the walk hits $\mathbb{D}$ in the same sub-segment $M_{j} \subset W_{k}$, and show it holds with only negligible probability.

Lemma 15. There exists $C>0$ such that for any $t>0, k>K^{\prime}(t)$,

$2^{k} \leq j<2^{k+1}$ and $x, y \in A_{j-1}$

$$
P^{x, y, j-1}\left(S R_{j}(0, t)\right) \leq \frac{C}{j^{6}} .
$$

Proof. Let $n_{0}$ be the first step $\mathbb{D}$ is hit in the segment $M_{j}$ at time 0 , and $n_{t}$ be the first step that $\mathbb{D}$ is hit in $M_{j}$ at time $t$, letting them equal $\infty$ if $\mathbb{D}$ is not hit in $M_{j}$ at all at that time. Notice that by symmetry, we can estimate

$$
\mathbf{P}^{x, y, j-1}\left(S R_{j}(0, t)\right) \leq 2 \mathbf{P}^{x, y, j-1}\left(S R_{j}(0, t) \cap\left(n_{0} \leq n_{t}\right)\right) .
$$

Let

and

$$
r_{1}=\frac{2^{(j-1)^{2}}}{2^{2(j+2)} j^{12}}
$$

$$
\tau_{r_{1}}=\min \left\{m>n_{0}: S_{m}(0)>r_{1}\right\}
$$

Denote by $L_{1}$ the event that $G_{j}(0)$ occurs, $\tau_{r_{1}}<\infty$ and $S_{m}(0) \neq(0,0)$ for all integers $m$ between $n_{0}$ and $\tau_{r_{1}}$.

Denote by $L_{2}$ the event that $G_{j}(t)$ occurs and $S_{m}(t) \neq(0,0)$ for all integers $m$ between $n_{t}$ and $s_{j}$.

Then

$$
S R_{j}(0) \cap G_{j}(0) \subseteq R_{j}(0) \cap L_{1}
$$

and

$$
S R_{j}(t) \cap G_{j}(t) \cap\left\{n_{0} \leq n_{t}\right\} \subseteq R_{j}(t) \cap L_{2} .
$$

Combining these we get

$$
\begin{aligned}
& S R_{j}(0, t) \cap G_{j}(0, t) \cap\left\{n_{0} \leq n_{t}\right\} \\
& \subset\left(S R_{j}(0) \cap G_{j}(0)\right) \cap\left(S R_{j}(t) \cap G_{j}(t) \cap\left\{n_{0} \leq n_{t}\right\}\right) \\
& \subset R_{j}(0) \cap L_{1} \cap R_{j}(t) \cap L_{2} .
\end{aligned}
$$


Thus we get

$$
\begin{aligned}
\mathbf{P}^{x, y, j-1}\left(S R_{j}(0, t)\right) \\
\leq 2 \mathbf{P}^{x, y, j-1}\left(S R_{j}(0, t) \cap\left\{n_{0} \leq n_{t}\right\}\right) \\
\leq 2 \mathbf{P}^{x, y, j-1}\left(S R_{j}(0, t) \cap G_{j}(0, t) \cap\left\{n_{0} \leq n_{t}\right\}\right)+2 \mathbf{P}^{x, y, j-1}\left(\left(G_{j}(0, t)\right)^{c}\right) \\
\leq 2 \mathbf{P}^{x, y, j-1}\left(R_{j}(0)\right) \cdot \mathbf{P}^{x, y, j-1}\left(L_{1} \mid R_{j}(0)\right) \cdot \mathbf{P}^{x, j-1}\left(R_{j}(t) \mid R_{j}(0) \cap L_{1}\right) \cdot \\
\quad \cdot \mathbf{P}^{x, y, j-1}\left(L_{2} \mid R_{j}(0, t) \cap L_{1}\right)+2 \mathbf{P}^{x, y, j-1}\left(\left(G_{j}(0, t)\right)^{c}\right) .
\end{aligned}
$$

To bound the probabilities of the five terms on the right hand side of (24) we now introduce six new events, bound the probabilities of these events and then use these bounds to bound the terms in (24).

Let $B_{1}$ be the event that

$$
n_{0}<s_{j-1}+\frac{2^{2(j-1)^{2}}}{j^{6}} .
$$

Recall that by Corollary 5, for any $M, N>0$, if $\left|S_{s_{j-1}(0)}\right|>M$, then

$$
\mathbf{P}\left(\exists s_{j-1}<n^{\prime}<s_{j-1}+N:\left|S_{n^{\prime}}(0)\right| \leq 1\right) \leq \frac{C N}{M^{2}} .
$$

Since for any $x \in A_{j-1}$ we have $|x| \geq 2^{(j-1)^{2}}$, we can set $M=2^{(j-1)^{2}}$ and $N=\frac{2^{2(j-1)^{2}}}{j^{6}}$ to conclude

$$
\begin{aligned}
\mathbf{P}^{x, y, j-1}\left(B_{1}\right) & =\mathbf{P}^{x, y, j-1}\left(\exists s_{j-1}<n^{\prime}<s_{j-1}+\frac{2^{2(j-1)^{2}}}{j^{12}}:\left|S_{n^{\prime}}(0)\right| \leq 1\right) \\
& \leq \frac{C \frac{2^{2(j-1)^{2}}}{j^{6}}}{\left(2^{(j-1)^{2}}\right)^{2}} \\
& \leq \frac{C}{j^{6}} .
\end{aligned}
$$

Let $I$ denote the set of all indices between $s_{j-1}$ and $s_{j-1}+\frac{2^{2(j-1)^{2}}}{j^{6}}$ for which, conditioned on our Poisson process, $X_{i}(0)$ and $X_{i}(t)$ are independent.

Let $B_{2}$ be the event that $|I|<\frac{2^{2(j-1)^{2}}}{2^{j+2} j^{6}}$.

If $B_{1}$ does not occur, then $|I|$ is the sum of at least $\frac{2^{2(j-1)^{2}}}{j^{6}}$ i.i.d. indicator variables each happening with probability

$$
p=1-e^{t}>\frac{1}{2} \min (1, t) \geq \frac{1}{2^{j+1}},
$$

(the last inequality holds because $j \geq 2^{k} \geq K(t) \geq|\log t|$ ). Therefore by monotonicity in $t$ and in the number of indicators, $|I|$ stochastically dominates the sum of $N=\frac{2^{2(j-1)^{2}}}{j^{6}}$ i.i.d. indicator variables $T_{i}$ each equaling 1 with probability $p=\frac{1}{2^{j+1}}$. Let $T=\sum_{1 \leq i \leq N} T_{i}$. 
As we are conditioning on $\left(B_{1}\right)^{c}$ we have that $|I|$ dominates $T$ and if $T \geq \frac{N P}{2}$ then

$$
|I| \geq \frac{N P}{2}=\frac{2^{2(j-1)^{2}}}{2^{j+2} j^{6}}
$$

and $B_{2}$ does not occur.

By the Chernoff bound

$$
\mathbf{P}\left(T<\frac{N p}{2}\right) \leq \mathbf{P}\left(|T-N p| \geq \frac{N p}{2}\right) \leq 2 e^{-c N p}
$$

for some absolute constant $c>0$. Thus

$$
\mathbf{P}^{x, y, j-1}\left(B_{2} \mid\left(B_{1}\right)^{c}\right) \leq \mathbf{P}\left(T>\frac{N p}{2}\right) \leq 2 e^{-\frac{c 2^{2(j-1)^{2}}}{2^{j+2} j^{6}}} \leq \frac{C}{j^{6}} .
$$

Put $r=\frac{2^{(j-1)^{2}}}{2^{j+2} j^{6}}$.

Let $B_{3}$ be the event that $\left|S_{n_{0}}(t)\right|<r$ and

$$
S_{n_{0}}(t)=\sum_{i<n_{0}} X_{i}(t)
$$

Rearranging the order of summation we can write

$$
S_{n_{0}}(t)=\sum_{i<n_{0}} X_{i}(t)=\sum_{i<n_{0}, i \notin I} X_{i}(t)+\sum_{i \in I} X_{i}(t)
$$

Put

$$
x=\sum_{i<n_{0}, i \notin I} X_{i}(t)
$$

Since all variables $\left\{X_{i}\right\}_{i} \in I$ have been re-rolled between time 0 and $t$, the probability that $\mid S_{n_{0}}(t)<$ $r \mid$ is the same as the probability that simple random walk starting at $x$ will be at distance less than $r$ from $(0,0)$ after $|I|$ steps. Therefore by the second part of Lemma 4

$$
\mathbf{P}^{x, y, j-1}\left(S_{n_{0}}(t)<\frac{\sqrt{|I|}}{j^{3}}\right) \leq \frac{C}{j^{6}}
$$

And since $\left(B_{2}\right)^{c}$ implies $|I|>\frac{2^{2(j-1)^{2}}}{2^{j+2} j^{6}}>r^{2} j^{6}$ we get

$$
\mathbf{P}^{x, y, j-1}\left(B_{3} \mid\left(B_{2}\right)^{c}\right)<\frac{C}{j^{6}}
$$

Next we bound the probability that $n_{t}-n_{0}$ is small. Let $B_{4}$ be the event that $0<n_{t}-n_{0}<\frac{r^{2}}{j^{6}}=$ $\frac{2^{2(j-1)^{2}}}{2^{j+2} j^{1} 2}$. Conditioning on $\left|S_{n_{0}}(t)\right|>r, B_{4}$ implies that the random walk at time $t$ gets to distance $r$ 
from its position at step $n_{0}$ in less than $\frac{r^{2}}{j^{6}}$ steps, therefore we can apply the first part of Lemma 4 to bound

$$
\mathbf{P}^{x, y, j-1}\left(B_{4} \mid\left(B_{3}\right)^{c}\right)<\frac{C}{j^{6}} .
$$

Let $I_{1}$ denote the set of all indices between $n_{0}$ and $n_{t}$ for which, conditioned on our Poisson process, $X_{i}(0)$ and $X_{i}(t)$ are independent. Let $B_{5}$ denote the event that $\left|I_{1}\right|<\frac{2^{2(j-1)^{2}}}{2^{4(j+2)} j^{12} 2}$. A similar calculation as done for $B_{2}$ shows that $\mathbf{P}^{x, y, j-1}\left(B_{5}\right)<\frac{C}{j^{6}}$

Let $B_{6}$ be the event that $\left|S_{n_{t}}(0)\right|<r_{1}$. Using Lemma 4 in a calculation similar to the one for $B_{3}$ gives that

$$
\mathbf{P}^{x, y, j-1}\left(B_{6} \mid\left(B_{5}\right)^{c}\right)<\frac{C}{j^{6}} .
$$

Thus we get

$$
\mathbf{P}^{x, y, j-1}\left(B_{i}\right) \leq \frac{C}{j^{6}} \quad i=1, \ldots, 6 .
$$

We will estimate the five probabilities in (24) as follows:

1. By Lemma 8,

$$
\mathbf{P}^{x, y, j-1}\left(R_{j}(0)\right)=\mathbf{P}^{x, j-1}\left(R_{j}(0)\right) \leq \frac{C}{j} .
$$

2. $L_{1}$ is included in the event that after step $n_{0}$, at for which $\left|S_{n_{0}}(0)\right|=1$, the walk reaches distance $r$ before hitting $(0,0)$, therefore by Lemma 3 ,

$$
\mathbf{P}^{x, y, j-1}\left(L_{1} \mid R_{j}(0)\right) \leq \frac{C}{\log r_{1}} \leq \frac{C}{j^{2}} .
$$

3. To estimate $\mathbf{P}^{x, y, j-1}\left(R_{j}(t) \mid R_{j}(0) \cap L_{1}\right)$, notice that $R_{j}(0)$ and $L_{1}$ depend only on what happens at time 0 , and $\left(B_{2}\right)^{c}$ implies $|I| \geq \frac{s_{j}}{2^{10 j}}$ (For all large enough $j$ ), so using Lemma 9 we get

$$
\mathbf{P}^{x, y, j-1}\left(R_{j}(t) \mid\left(B^{2}\right)^{c} \cap R_{j}(0) \cap L_{1}\right) \leq \frac{C}{j},
$$

and therefore

$$
\mathbf{P}^{x, y, j-1}\left(R_{j}(t) \mid R_{j}(0) \cap L_{1}\right) \leq \frac{C}{j}+\frac{C}{j^{6}} \leq \frac{C}{j} .
$$

4. To estimate $\mathbf{P}^{x, y, j-1}\left(L_{2} \mid R_{j}(0, t) \cap L_{1}\right)$, we partition according to the value of $n_{t}$. Since for a given $n_{t}$, the event $L_{2}$ is independent of the variables $\left\{X_{i}(0)\right\}_{i<n_{t}} \cup\left\{X_{i}(t)\right\}_{i<n_{t}}$, and the event $R_{j}(0, t) \cap L_{1} \cap\left(B_{6}\right)^{c}$ depend only on these variables, we have $\forall N_{t}$

$$
\mathbf{P}^{x, y, j-1}\left(L_{2} \mid R_{j}(0, t) \cap L_{1} \cap\left(B_{6}\right)^{c} \cap\left(n_{t}=N_{t}\right)\right)=\mathbf{P}^{x, y, j-1}\left(L_{2} \mid n_{t}=N_{t}\right) .
$$

Since by Lemma 3 ,

$$
\mathbf{P}^{x, y, j-1}\left(L_{2} \mid n_{t}=N_{t}\right) \leq \frac{C}{j^{2}}
$$


for any value of $N_{t}$, we deduce that

$$
\mathbf{P}^{x, y, j-1}\left(L_{2} \mid R_{j}(0, t) \cap L_{1} \cap\left(B_{6}\right)^{c}\right) \leq \frac{C}{j^{2}}
$$

and therefore

$$
\mathbf{P}^{x, y, j-1}\left(L_{2} \mid R_{j}(0, t) \cap L_{1}\right) \leq \frac{C}{j^{2}}+\mathbf{P}^{x, y, j-1}\left(B_{6}\right) \leq \frac{C}{j^{2}} .
$$

5. Last we use Lemma 6 to bound

$$
\mathbf{P}^{x, y, j-1}\left(\left(G_{j}(0, t)\right)^{c}\right) \leq 2 \mathbf{P}^{x, j-1}\left(\left(G_{j}(0)\right)^{c}\right) \leq \frac{C}{j^{10}} .
$$

Combining (32), (33), (34), (35) and (36) into (24) we get

$$
\begin{aligned}
\mathbf{P}^{x, y, j-1} & \left(S R_{j}(0, t)\right) \\
\leq & 2 \mathbf{P}^{x, y, j-1}\left(R_{j}(0)\right) \cdot \mathbf{P}^{x, y, j-1}\left(L_{1} \mid R_{j}(0)\right) \\
& \cdot \mathbf{P}^{x, y, j-1}\left(R_{j}(t) \mid R_{j}(0) \cap L_{1}\right) \cdot \mathbf{P}^{x, y, j-1}\left(L_{2} \mid R_{j}(0, t) \cap L_{1}\right) \\
& +\mathbf{P}^{x, y, j-1}\left(\left(G_{j}(0, t)\right)^{c}\right) \\
\leq & 2 \frac{C}{j} \cdot \frac{C}{j^{2}} \cdot \frac{C}{j} \cdot \frac{C}{j^{2}}+\frac{C}{j^{10}} \\
\leq & \frac{C}{j^{6}} .
\end{aligned}
$$

Lemma 16. There exists a constant $C>0$ s.t. for any $t>0$ and any $k>K^{\prime}(t)$

$$
\mathbf{P}\left(S E_{k}(0, t) \mid S E_{k-1}(0, t)\right) \leq\left(\beta_{k} \pi \sum_{2^{k} \leq j<2^{k+1}} \frac{1}{j^{3}}\right)^{2}+\frac{C k}{2^{5 k}} .
$$

Proof. $S E_{k-1}(0, t) \subseteq G_{2^{k}-1}(0, t)$, so by the Markov property of simple random walk we have

$$
\mathbf{P}\left(S E_{k}(0, t) \mid S E_{k-1}(0, t)\right) \leq \max _{x, y \in A_{2^{k}-1}} \mathbf{P}^{x, y, 2^{k}-1}\left(S E_{k}(0, t)\right) .
$$

$S E_{k}(0, t)$ is the disjoint union of the events $S E_{k}^{j_{1}, j_{2}}(0, t)$, therefore

$$
\begin{aligned}
\max _{x, y \in A_{2^{k}-1}} \mathbf{P}^{x, y, 2^{k}-1}( & \left.S E_{k}(0, t)\right) \\
\leq & \sum_{2^{k} \leq j_{1}, j_{2}<2^{k+1}} \max _{x, y \in A_{2^{k}-1}} \mathbf{P}^{x, y, 2^{k}-1}\left(S E_{k}^{j_{1}, j_{2}}(0, t)\right) \\
= & \sum_{2^{k} \leq j_{1} \neq j_{2}<2^{k+1}} \max _{x, y \in A_{2^{k}-1}} \mathbf{P}^{x, y, 2^{k}-1}\left(S E_{k}^{j_{1}, j_{2}}(0, t)\right) \\
& +\sum_{2^{k} \leq j \leq 2^{k+1}} \max _{x, y \in A_{2^{k}-1}} \mathbf{P}^{x, y, 2^{k}-1}\left(S E_{k}^{j, j}(0, t)\right) .
\end{aligned}
$$


By Lemma 14, for any $2^{k} \leq j_{1} \neq j_{2}<2^{k+1}$

$$
\max _{x, y \in A_{2^{k}-1}} \mathbf{P}^{x, y, 2^{k}-1}\left(S E_{k}^{j_{1}, j_{2}}(0, t)\right) \leq \beta_{k}^{2} \frac{\pi}{j_{1}^{3}} \frac{\pi}{j_{2}^{3}}+\frac{C k}{2^{7 k}} .
$$

$S E_{k}^{j, j}(0, t) \subseteq S R_{j}(0, t)$, therefore by Lemma 15 , for any $2^{k} \leq j<2^{k+1}$

$$
\max _{x, y \in A_{2^{k}-1}} \mathbf{P}^{x, y, 2^{k}-1}\left(S E_{k}^{j, j}(0, t)\right) \leq \frac{C}{j^{6}} .
$$

Combining (38) and (39) into (37) we conclude

$$
\begin{aligned}
\max _{x, y \in A_{2^{k}-1}} \mathbf{P}^{x, y, 2^{k}-1}\left(S E_{k}(0, t)\right) \\
\leq \beta_{k}^{2} \pi^{2} \sum_{2^{k} \leq j_{1} \neq j_{2}<2^{k+1}}\left(\frac{1}{j_{1}^{3}} \frac{1}{j_{2}^{3}}+\frac{C k}{2^{7 k}}\right)+\sum_{2^{k} \leq j<2^{k+1}} \frac{C}{j^{6}} \\
\leq\left(\beta_{k} \pi \sum_{2^{k} \leq j<2^{k+1}} \frac{1}{j^{3}}\right)^{2}+\frac{C k}{2^{5 k}}
\end{aligned}
$$

\subsection{Proof of Theorem 2}

Proof of Theorem 2. Define

$$
f(t, M)=\frac{\mathbf{P}\left(\bigcap_{1 \leq k \leq M} S E_{k}(0, t)\right)}{\left(\mathbf{P} \bigcap_{1 \leq k \leq M}\left(S E_{k}(0)\right)\right)^{2}} .
$$

Set $k_{0}=K^{\prime}(t)$. Then

$$
f(t, M) \leq \frac{1}{\mathbf{P}\left(S E_{k_{0}}(0)\right)^{2}} \prod_{k=k_{0}+1}^{M} \frac{\mathbf{P}\left(S E_{k}(0, t) \mid S E_{k-1}(0, t)\right)}{\mathbf{P}\left(S E_{k}(0) \mid S E_{k-1}(0)\right)^{2}} .
$$

We start by estimating the first factor:

$$
\frac{1}{\mathbf{P}\left(S E_{k_{0}}(0)\right)^{2}} \leq C \prod_{0<k \leq k_{0}} \frac{1}{\mathbf{P}\left(S E_{k}(0) \mid S E_{k-1}(0)\right)^{2}} .
$$


Using the lower bound from Lemma 13 we get that this is bounded above by

$$
\begin{aligned}
\frac{1}{\mathbf{P}\left(S E_{k_{0}}(0)\right)^{2}} & \leq C \prod_{0<k \leq k_{0}} \frac{1}{\left(\left(\beta_{k} \pi \sum_{2^{k} \leq j<2^{k+1}} \frac{1}{j^{3}}\right)-\frac{C k}{2^{3 k}}\right)^{2}} \\
& \leq C \prod_{0<k \leq k_{0}} \frac{1}{\left(\frac{C}{2^{2 k}}-\frac{C k}{2^{3 k}}\right)^{2}} \\
& \leq C \prod_{0<k \leq k_{0}} C 2^{4 k} \\
& \leq C^{k_{0}} 2^{2 k_{0}^{2}} \\
& \leq C 2^{3 k_{0}^{2}} .
\end{aligned}
$$

We now recall that we chose $k_{0}$ to be the smallest integer larger then $\log (|\log t|+1)+1$, to get

$$
\begin{aligned}
\frac{1}{\mathbf{P}\left(S E_{k_{0}}(0)\right)^{2}} & \leq C 2^{3 k_{0}^{2}} \\
& \leq C\left(2^{k_{0}}\right)^{3 k_{0}} \\
& \leq C\left(2^{\log (|\log t|+1)+2}\right)^{3 k_{0}} \\
& \leq C(4(1+|\log t|))^{3(\log (|\log t|+1)+2)}
\end{aligned}
$$

For the second factor of (40), we use Lemma 13 to bound the denominator, together with Lemma 16 to bound the numerator. We get

$$
\begin{aligned}
\prod_{k=k_{0}+1}^{n} \frac{\mathbf{P}\left(S E_{k}(0, t) \mid S E_{k-1}(0, t)\right)}{\mathbf{P}\left(S E_{k}(0) \mid S E_{k-1}(0)\right)^{2}} & \leq \prod_{k=k_{0}+1}^{n} \frac{\left(\beta_{k} \pi \sum_{2^{k} \leq j<2^{k+1}} \frac{1}{j^{3}}\right)^{2}+\frac{C k}{2^{5 k}}}{\left(\beta_{k} \pi \sum_{2^{k} \leq j<2^{k+1}} \frac{1}{j^{3}}-\frac{C k}{2^{3 k}}\right)^{2}} \\
& \leq \prod_{k=k_{0}+1}^{n} \frac{\left(\beta_{k} \pi \sum_{2^{k} \leq j<2^{k+1}} \frac{1}{j^{3}}\right)^{2}+\frac{C k}{2^{5 k}}}{\left(\beta_{k} \pi \sum_{2^{k} \leq j<2^{k+1}} \frac{1}{j^{3}}\right)^{2}-\frac{C k}{2^{5 k}}} \\
& \leq \prod_{k=k_{0}+1}^{n}\left(1+\frac{C k}{2^{k}}\right) \\
& \leq C .
\end{aligned}
$$

Multiplying the two estimates we deduce that

$$
f(t, M) \leq C(4(1+|\log t|))^{3(\log (|\log t|+1)+2)}
$$

for any $M$. Since

$$
\int_{0}^{1} C(4(1+|\log t|))^{3(\log (|\log t|+1))+2} d t<\infty
$$

the proof is complete. 


\section{Proof of Theorem 1}

The proof of Theorem 1 follows from Theorem 2 and a second moment argument exactly as in [5]. We give it here for the sake of completeness.

Proof of Theorem 1. Define

$$
\begin{gathered}
E_{M}(t)=\bigcap_{1 \leq i \leq M} S E_{i}(t) \\
T_{M}=\left\{t: t \in[0,1] \text { and } E_{M}(t) \text { occurs }\right\}
\end{gathered}
$$

and

$$
T=\cap_{1}^{\infty} \overline{T_{M}} \text {. }
$$

Now we show that $T$ is contained in the union of $S^{\text {exc }}$ and the countable set

$$
\Lambda=\left(\cup_{n, m} \tau_{n}^{(m)}\right) \cup 1
$$

If $t \in \cap_{1}^{\infty} T_{M}$ then $t \in S^{\text {exc }}$. So if $t \in T \backslash S^{\text {exc }}$ then $t$ is contained in the boundary of $T_{M}$ for some $M$. For any $M$ the boundary of $T_{M}$ is contained in $\Lambda$. Thus if $t \in T \backslash S^{\operatorname{exc}}$ then $t \in \Lambda$ and

$$
T \subset S^{\text {exc }} \cup \Lambda \text {. }
$$

As $\Lambda$ is countable if $T$ has dimension one with positive probability then so does $S^{\text {exc }}$.

By Theorem 2 there exists $f(t)$ such that

$$
\int_{0}^{1} f(t) d t<\infty
$$

and for all $M$

$$
\frac{\mathbf{P}\left(E_{M}(0, t)\right)}{\left(\mathbf{P}\left(E_{M}(0)\right)\right)^{2}}<f(M, t)<f(t) .
$$

Let $\mathscr{L}(*)$ denote Lebesgue measure on $[0,1]$. Then we get

$$
\begin{aligned}
\mathbf{E}\left(\mathscr{L}\left(T_{M}\right)^{2}\right) & =\int_{0}^{1} \int_{0}^{1} \mathbf{P}\left(E_{M}(r, s)\right) d r \times d s \\
& =\int_{0}^{1} \int_{0}^{1} \mathbf{P}\left(E_{M}(0,|s-r|)\right) d r \times d s \\
& \leq \int_{0}^{1} 2 \int_{0}^{1} \mathbf{P}\left(E_{M}(0, t)\right) d t \times d s \\
& \leq 2 \int_{0}^{1} f(t) \mathbf{P}\left(E_{M}(0)\right)^{2} d t \\
& \leq 2 \mathbf{P}\left(E_{M}(0)\right)^{2} \int_{0}^{1} f(t) d t .
\end{aligned}
$$


The equality (44) is true by Fubini's theorem, (45) is true because

$$
E_{M}(a, b)=E_{M}(b, a)=E_{M}(0,|b-a|)
$$

and (46) follows from (43).

By Jensen's inequality if $h(x)=0$ for all $x \notin A$ then

$$
\mathbf{E}\left(h^{2}\right) \geq \frac{\mathbf{E}(h)^{2}}{\mathbf{P}(A)} .
$$

Then we get

$$
\begin{aligned}
2 \mathbf{P}\left(E_{M}(0)\right)^{2} \int_{0}^{1} f(t) d t & \geq \mathbf{E}\left(\mathscr{L}\left(T_{M}\right)^{2}\right) \\
& \geq \frac{\mathbf{E}\left(\mathscr{L}\left(T_{M}\right)\right)^{2}}{\mathbf{P}\left(T_{M} \neq \emptyset\right)} \\
& \geq \frac{\mathbf{P}\left(E_{M}(0)\right)^{2}}{\mathbf{P}\left(T_{M} \neq \emptyset\right)}
\end{aligned}
$$

where (49) is a restatement of (47), and (50) follows from (48) with $\mathscr{L}\left(T_{M}\right)$ and $T_{M} \neq \emptyset$ in place of $h$ and $A$.

Thus for all $M$

$$
\mathbf{P}\left(T_{M} \neq \emptyset\right) \geq \frac{1}{2 \int_{0}^{1} f(t) d t}>0 .
$$

As $T$ is the intersection of the nested sequence of compact sets $\bar{T}_{M}$

$$
\mathbf{P}(T \neq \emptyset)=\lim _{M \rightarrow \infty} \mathbf{P}\left(\bar{T}_{M} \neq \emptyset\right)=\lim _{M \rightarrow \infty} \mathbf{P}\left(T_{M} \neq \emptyset\right) \geq \frac{1}{2 \int_{0}^{1} f(t) d t} .
$$

Now we show that the dimensions of $T$ and $S^{\text {exc }}$ are one. By Lemma 5.1 of [11] for any $\beta<1$ there exists a random nested sequence of compact sets $F_{k} \subset[0,1]$ such that

$$
\mathbf{P}\left(r \in F_{k}\right) \geq C\left(s_{k}\right)^{-\beta}
$$

and

$$
\mathbf{P}\left(r, t \in F_{k}\right) \leq C\left(s_{k}\right)^{-2 \beta}|r-t|^{-\beta} .
$$

These sets also have the property that for any set $T$ if

$$
\mathbf{P}\left(T \cap\left(\cap_{1}^{\infty} F_{k}\right) \neq \emptyset\right)>0
$$

then $T$ has dimension at least $\beta$. We construct $F_{k}$ to be independent of the dynamical random walk. So by (42), (51) and (52) we get

$$
\frac{\mathbf{P}\left(r, t \in T_{M} \cap F_{M}\right)}{\mathbf{P}\left(r \in T_{M} \cap F_{M}\right)^{2}} \leq C(1+|\log | r-t||)^{3|\log (|\log | r-t||+1)|}|r-t|^{-\beta} .
$$


Since

$$
\int_{0}^{1} C(1+|\log t|)^{3|\log (|\log t|+1)|} t^{-\beta} d t<\infty,
$$

the same second moment argument as above and (54) implies that with positive probability $T$ satisfies (53). Thus $T$ has dimension $\beta$ with positive probability. As

$$
T \subset\left(S^{\operatorname{exc}} \cap[0,1]\right) \cup \Lambda,
$$

and $\Lambda$ is countable, the dimension of the set of $S^{\operatorname{exc}} \cap[0,1]$ is at least $\beta$ with positive probability. By the ergodic theorem the dimension of the set of $S^{\operatorname{exc}}$ is at least $\beta$ with probability one. As this holds for all $\beta<1$ the dimension of $S^{\text {exc }}$ is one a.s.

\subsection{Extending the technique}

We end the paper with three remarks on the scope of the techniques in this paper.

1. The techniques in this paper can be extended to show other sets of exceptional times exist. For any two finite sets $E$ and $V$, for which it is possible to visit every point in $V$ without hitting $E$, there is a.s. an exceptional time $t$ such that each point in $V$ is visited infinitely often, while the set $E$ is not visited at all.

2. Not every set can be avoided by the random walk, as shown in the following claim:

Claim 17. Let $L$ be a subset of $\mathbb{Z}^{2}$, with the property that there exits some $M>0$, such that every point in $\mathbb{Z}^{2}$ is at distance at most $M$ from some point in $L$. Then

$$
\mathbf{P}\left(\exists t \in[0,1]:\left|\left\{n: S_{n}(t) \notin L\right\}\right|<\infty\right)=0
$$

i.e there are a.s. no exceptional times at which $L$ is visited only finitely often.

Proof. First, we can reduce to the case that there is some (possible) path from 0 that misses $L$. Otherwise we can just drop points out of $L$ until such a path exists without ruining the desired condition on $L$

Second, we note that it is enough to prove that there are a.s. no times at which the random walk never hits $L$. This follows from the Markov property of random walk, and the fact that changing finitely many moves is enough to make a walk that visits $L$ a finite number of times miss $L$ all together.

Let

$$
Q_{n}=\left\{t \in[0,1]: S_{i}(t) \notin L \quad 0 \leq \forall i \leq n\right\} .
$$

Then an exceptional time exists if and only if

$$
\bigcap_{n \geq 0} Q_{n} \neq \emptyset
$$


The condition on $L$ ensures that there is some $\epsilon>0$, such that for any simple random walk on $Z^{2}$, regardless of current position or history, there is a probability of at least $\epsilon$ of hitting $L$ in the next $2 M$ steps. Therefore there exists some constants $A>0$ and $0<c<1$ such that

$$
\mathbf{P}\left(0 \in Q_{n}\right) \leq A c^{n}
$$

For any $i \geq 0$ define

$$
m_{i}=\min \left\{m \geq 0: \tau_{i}^{(m)}>1\right\}
$$

Then

$$
R E_{i}=\left\{\tau_{i}^{(j)}\right\}_{j=0}^{m_{i}}
$$

is the set of times in $[0,1]$ in which the $i-t h$ move gets resampled. Let $H_{n}=\bigcup_{i=0}^{n} R E_{i}$. Then $H_{n}$ is the set of all times at which one of the first $n$ moves of the walk are re-sampled, and if we order $H_{n}$, then between two consecutive times in $H_{n}$, the first $n$ steps of the walk do not change. Thus $Q_{n} \neq \emptyset$ if and only if there exists some $\tau \in H_{n}$ for which $\tau \in Q_{n}$. Since $\left\{S_{n}(t)\right\}$ behaves like a simple random walk for all $t$, we have, by linearity of expectation

$$
\mathbb{E}\left(\# \tau \in H_{n}: \tau \in Q_{n}\right)=\mathbb{E}\left(\left|H_{n}\right|\right) \mathbf{P}\left(0 \in Q_{n}\right) \leq 2 A n c^{-n}
$$

Thus

$$
\mathbf{P}\left(Q_{n} \neq \emptyset\right) \leq \mathbb{E}\left(\# \tau \in H_{n}: \tau \in Q_{n}\right) \leq 2 A n c^{-n}
$$

And consequently

$$
\mathbf{P}\left(\bigcap_{n \geq 0} Q_{n} \neq \emptyset\right)=0
$$

So no exceptional times exist.

3. We finish with an open question:

Is there a set $A \subset \mathbb{Z}^{2}$ such that both $A$ and $A^{c}$ are infinite and almost surely there are exceptional times in which every element of $A$ is hit finitely often and every element of $A^{c}$ is hit infinitely often?

\section{References}

[1] Adelman, O., Burdzy, K. and Pemantle, R. (1998). Sets avoided by Brownian motion. Ann. Probab. 26 429-464. MR1626170

[2] Benjamini, Itai; Häggström, Olle; Peres, Yuval; Steif, Jeffrey E. Which properties of a random sequence are dynamically sensitive? Ann. Probab. 31 (2003), no. 1, 1-34. MR1959784

[3] Fukushima, Masatoshi. Basic properties of Brownian motion and a capacity on the Wiener space. J. Math. Soc. Japan 36 (1984), no. 1, 161-176. MR0723601

[4] Häggström, O., Peres, Y. and Steif, J. E. (1997). Dynamical percolation. Ann. Inst. H. Poincaré Probab. Statist. 33 497-528. MR1465800 
[5] Hoffman C. Recurrence of Simple Random Walk on $\mathbb{Z}^{2}$ is Dynamically Sensitive ALEA 1 35-45. MR2235173

[6] Kozma G. and Schreiber E. An asymptotic expansion for the discrete harmonic potential. Electron. J. Probab. 9 (2004), no. 1, 1-17 math.PR/0212156 MR2041826

[7] Lawler, Gregory F. Intersections of random walks. Probability and its Applications. Birkhäuser Boston, Inc., Boston, MA, 1991.

[8] Levin, D., Khoshnevesian D. and Mendez, P. Exceptional Times and Invariance for Dynamical Random Walks. math.PR/0409479 to appear in Probability Theory and Related Fields. MR2226886

[9] Levin, D., Khoshnevesian D. and Mendez, P. On Dynamical Gaussian Random Walks. math.PR/0307346 to appear in Annals of Probability.

[10] Penrose, M. D. On the existence of self-intersections for quasi-every Brownian path in space. Ann. Probab. 17 (1989), no. 2, 482-502. MR0985374

[11] Peres, Yuval. Intersection-equivalence of Brownian paths and certain branching processes. Comm. Math. Phys. 177 (1996), no. 2, 417-434. MR1384142

[12] Spitzer, Frank. Principles of random walks. Second edition. Graduate Texts in Mathematics, Vol. 34. Springer-Verlag, New York-Heidelberg, 1976. MR0388547 